Review

\title{
Genetic, Social and Behavioral Risk Factors Associated with Alzheimer's Disease
}

Vineela Nagamalla ${ }^{1}$, Jay Patel ${ }^{2}$, Mir Saleem ${ }^{3,}{ }^{*}$

1. Albert Einstein College of Medicine, 1300 Morris Park Ave, Bronx, NY, USA; E-Mail: vn166@mynsu.nova.edu

2. Lake Erie College of Osteopathic Medicine, 1858 W Grandview Blvd, Erie, PA, USA; E-Mail: ip2529@mynsu.nova.edu

3. Dept. of Biological Sciences, $3^{\text {rd }}$ Floor, Parker Building, 3301 College Avenue, Halmos College of Art and Sciences, NOVA Southeastern University, Fort Lauderdale, FL 33314, USA; E-Mail: msaleem@nova.edu

* Correspondence: Mir Saleem; E-Mail: msaleem@nova.edu

Academic Editor: Satya V. V. N. Kothapalli

Special Issue: $\underline{\text { Roles of Ultrasound in Alzheimer's Disease Diagnosis and Therapy }}$

OBM Neurobiology

2021, volume 5, issue 3

doi:10.21926/obm.neurobiol.2103104
Received: December 20, 2020

Accepted: July 13, 2021

Published: July 21, 2021

\begin{abstract}
Alzheimer's disease (AD) is a neurodegenerative condition which consists of stages of mental, memory, and cognitive decline. As it continues to stand as the sixth leading cause of death in the U.S., 44 million people worldwide are affected. The objective of this paper is to explore the risk factors for $A D$ in an attempt to examine potential causes for the incidence of $A D$. We examined genetics, lifestyle, and pre-existing conditions as risk factors for AD. We found that there are various alleles, such as APP, PSEN, ADAM, APOE, and TREM2, that play a role in the development of $A D$. It was also determined that behavioral modifications such as following a Mediterranean and ketogenic diet, engaging in regular intellectual activities, and participating in regular physical
\end{abstract}

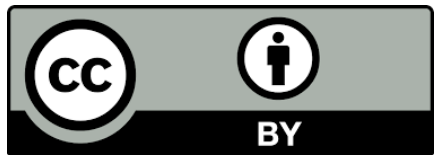

(C) 2021 by the author. This is an open access article distributed under the conditions of the Creative Commons by Attribution License, which permits unrestricted use, distribution, and reproduction in any medium or format, provided the original work is correctly cited. 
exercise can all reduce the risk of developing AD. The comorbidity of existing conditions such as hypertension, diabetes mellitus, high cholesterol, and cardiovascular diseases all had an impact on developing $A D$. There is evidence that genetics, lifestyle, and pre-existing conditions significantly impact the incidence of $A D$; however, understanding how all these risk factors interrelate with each other is a recommended topic for further study.

\section{Keywords}

Alzheimer's Disease; neurodegenerative disorder; risk factors; genetics; lifestyle; pre-existing conditions

\section{Introduction}

Alzheimer's disease (AD) is a progressive neurological condition that causes deterioration in memory, function, and behavior. It typically begins and remains in an asymptomatic phase for a long period of time, followed by symptomatic pre-dementia phase and then can transition to the dementia stage. It is estimated that 5.8 million Americans are affected by this disease in 2019. Health-care costs for AD has been estimated to be about $\$ 172$ billion per year [1].

This neurodegenerative disease is often characterized by mental decline, memory loss, confusion, disorientation, and etc. along with possible behavioral, mood, and/or psychological changes. Neuropathological hallmarks of $A D$ are identified as extracellular depositions of amyloid- $\beta(A \beta)$ peptide in neuritic plaques and neurofibrillary tangles. Some changes such as microgliosis or loss of white matter and neurons can also occur. However, mechanisms behind many of these processes are still unknown. As Alzheimer's remains as the sixth leading cause of death in the U.S., there is a need for more research analyzing prevention, early detection, reversal, and treatments options [2].

This paper examines various risk factors playing a role in the incidence of Alzheimer's disease. It is important to investigate risk factors as it may open new doors in understanding Alzheimer's disease and allow for preventive and treatable measures to be taken. This paper evaluated the impact that genetics, social and behavioral factors, and pre-existing conditions can have on the incidence of Alzheimer's disease.

\section{Genetics}

\subsection{Deterministic Genes}

\subsubsection{APP, PSEN1, PSEN2}

Early onset of Alzheimer's can be caused by mutations to the transmembrane neuronal protein, $\beta$ amyloid precursor protein (APP). Cleavage of APP by $\beta$-secretase and $\psi$-secretase results in amyloid- $\beta$ peptide $(A \beta)$. The amyloid cascade hypothesis states that mutations in APP or alterations in A $\beta$ homeostasis lead to an accumulation of $A \beta$ and plaques, which can be enough to trigger pathological 
symptoms of Alzheimer's. However, cleavage of APP by $\alpha$-secretase shows neuroprotective effects as it generates nonamyloidogenic fragments. Bioinformatic analysis show that rare variants of $A P P$, such as APP N660Y, can increase the risk of Alzheimer's while APP A673T can decrease and APP E599K can show no effect on the risk of developing Alzheimer's [3].

Previous genome-wide association studies have found susceptibility loci, such as CLU, B1N1, and CR1, that affect processes involving APP in non-Hispanic Whites [4].

$C L U$ is also known as apolipoprotein $\mathrm{J}$ and mediates lipid transport. It is thought that $C L U$ can affect $A \beta$-aggregation and $A \beta$ clearance via endocytosis by acting as an extracellular chaperone. B1N1 is part of the Bin1/amphiphysin/RVS167 genes, which are involved in various cellular processes including membrane trafficking and clathrin-mediated endocytosis. Since this family of genes are involved in membrane transport and actin dynamics, $B 1 N 1$ can affect processing, production or clearance of $A \beta$. Additionally, CR1 is a cell receptor that participates in the complement system by binding to complement factors $\mathrm{C} 3 \mathrm{~b}$ and $\mathrm{C} 4 \mathrm{~b}$. It is found that $\mathrm{A} \beta$ oligomers bind to $\mathrm{C} 3 \mathrm{~b}$ so $\mathrm{CR} 1$ can possibly allow clearance of $A \beta$. As part of the complement system, CR1 is also involved in neuroinflammation, which is a characteristic of Alzheimer's disease. However, CLU can also be an inhibitor in the process. This first set of loci are involved in the metabolism, processing, or endocytosis of APP [5-7].

Additionally, $A B C A 7$ is another identified susceptibility gene that is involved in the metabolism and processing of APP. Part of the ABC family proteins, ABCA7 is a transmembrane ATP-binding cassette transporter which controls the biogenesis of high-density lipoprotein and transport of important proteins, such as amyloid precursor protein. In genome-wide association studies in African Americans, $A B C A 7$ is a major susceptibility locus and had a greater risk factor than compared to Caucasians. This locus has a $70-80 \%$ increase in risk in African Americans while 10-20\% increase in risk in Caucasians. However, further studies in African Americans have to take place to confirm this as a target for prevention, testing, and treatment for AD. In addition to genetic origins, studies have shown that traumatic brain injury can vary the $A \beta$ and tau pathologies in the brain. Changes such as elevated levels of $A \beta$ and overproduction of APP in the cerebrospinal fluid can occur $[8,9]$.

Mutations within presenilin (PSEN) 1 and PSEN2 increase $\gamma$-secretases leading to a buildup of amyloid- $\beta$. Additionally, mutations in APP, Presenilin (PSEN) 1 and PSEN2 have also been linked to cause early onset $A D(E O A D)[10,11]$.

$A P P, P S E N 1$ and PSEN2 genes are all inherited autosomal dominant, and mutations to these $A D$ genes have been found with high levels of $A \beta$ aggregation and start of Alzheimer's disease. As they are involved in the EOAD, they can also be used as biomarkers [4].

Recent research points out that PSEN1 and PSEN2 mutations has been associated with late onset AD (LOAD) as well but are unlikely to have a significant contribution towards the susceptibility of LOAD [11].

\subsection{APP-Modifying Genes}

\subsubsection{ADAM Family}

The ADAM (A disintegrin and metalloprotease) family is responsible for controlling $\alpha$-secretase activity [12]. The enzyme $\alpha$-secretase is responsible for cleaving APP in the middle of the A $\beta$ domain 
[13]. There are three members of the ADAM family: ADAM9, ADAM10, ADAM17. Each member of this family has a unique function [12]. ADAM9 is suggested to have an indirect role in modulating $\alpha$ secretase cleavage. Unlike ADAM10, ADAM17 is not directly involved in the coordinated expression of APP in the human brain. ADAM17 is required for phorbol myristate acetate (PMA) to increase $\alpha$ secretase cleavage [14]. ADAM 10 is a type I membrane protein and is most notably known for its combined ability to regulate and constitute $\alpha$-secretase activity along with being responsible for coordinating mRNA expression of APP in human neurons [12].

Patients with sporadic Alzheimer's patients have been reported to have decreased levels of ADAM10 and SAPP $\alpha$ levels. Moreover, it has been observed that mice with excess ADAM10 expression have elevated SAPP $\alpha$ levels and reduced A $\beta$ formation [12]. The ADAM10 promoter region contains a transcriptional binding site that is responsive to retinoic acid. Retinoic acid along with other vitamin $A$ derivatives, in theory, should increase the expression of ADAM10, which will in turn elevate APP $\alpha$ secretase cleavage and reduce $A \beta$ plaque accumulations. This points at retinoic acid as a possible drug that could be tested in the future to determine if it would have an effect on AD [14].

The Q170H and R181G mutations in ADAM10 sharply decreases the $\alpha$-secretase activity of ADAM10 further leading to increases in $A \beta$ plaques. Q170H and R181G mutations have been partially penetrant in familial LOAD. The mutations affecting late onset instead of early onset draws attention to the small effect $A \beta$ plaque accumulation plays in LOAD compared to EOAD mutations in APP, PSEN1, and PSEN2 [12].

\subsection{Risk Genes}

\subsubsection{APOE}

The $A P O E$ allele, which produces the apolipoprotein $\mathrm{E}$, have been identified as a major genetic risk factor for AD. APOE, a lipid transport protein, is present in plasma and in the brain. It is secreted by glia, and as a result it is also present in the CSF. Although the mechanism is not clear, there is evidence that shows APOE can affect neural plasticity and structure which can further the progression of AD. It is hypothesized that APOE can interact with and influence the clearance and deposition of amyloid- $\beta$ peptide. Studies in vivo have shown that APOE can colocalize with $A \beta$ in the brains of Alzheimer's disease. While studies in vitro have shown that through an isoform specific manner, APOE binds to A $\beta$ and can affect $A \beta$ fibril formation. However, other factors such as the lipidation state of APOE and the presence of binding proteins can affect these results, thus causing difficulty in understanding this process [15].

For more than ten years, it had been thought the APOEE4 allele on chromosome 19q13 causes susceptibility to $A D$ in non-Hispanics Whites. Three alleles $\varepsilon 2, \varepsilon 3$, and $\varepsilon 4$ produces three isoforms of APOE. Receiving one copy of the APOE\&4 allele causes a two or three-fold increased risk, while receiving two copies of the APOE\&4 allele causes a five or more-fold increase in risk. In addition, each copy lowers the age at which disease starts by six to seven years [2]. In general, there is a correlation between increasing the gene dose of APOE\&4 allele and the increased number of $A \beta$ in AD patients. For instance, studies using transgenic mice that express mouse APOE4 or human APOE3 or APOE4 were used to understand the $A P O E / A \beta$ interactions in the pathogenesis of Alzheimer's disease. To understand the 
role of APOE/A $\beta$ in the pathogenesis of Alzheimer's disease, expression of APOE4 gene in mice and APOE3 and APOE4 genes in human were studied. It was found that human APOE isoforms had suppressed deposition of $A \beta$ for 6 or more months longer, and there were reduced levels of total $A \beta$ in the hippocampus compared to the mice of mouse APOE. However, following the inhibition of deposition in human APOE expressing mice, accumulation of $A \beta$ resulted in $A \beta$ fibrils and plaques. The study also found that by 15 months of age, $89 \%$ of the APOE 4 mice had deposits of $A \beta$ in the hippocampus compared to only $39 \%$ of APOE3 mice. As a result, APOE4 poses a greater A $\beta$ burden in terms of $A \beta$ fibrils and neuritic plaque compared to APOE3 [16].

Although $A P O E \varepsilon 4$ can correlate to low levels of cognitive performance in terms of memory, the presence of the allele is not required nor enough to cause the development of $A D$. The inconsistent association between the $\varepsilon 4$ and late onset $A D$ was seen in other ethnic groups, those that are not nonHispanics Whites. On the other hand, research identified $A P O E$ as a susceptibility gene in African Americans, while this finding was inconsistent across previous studies [4].

Additional factors, such as traumatic head injury, when coupled with the APOEE4 allele can have synergistic effects on the incidence of $A D$. For instance, when there was a history of head injury with APOE4 in the elderly person, the risk of $A D$ increased by 10 -fold compared to the two-fold increase with APOE4 alone or to the absence of risk with head injury alone [17].

\subsubsection{TREM2}

The TREM2 receptor on microglia is utilized for phagocytosis and inflammation repression. A rare missense mutation in the TREM2 gene is associated with an increase in the risk of late onset Alzheimer's disease. The variant $\mathrm{R} 47 \mathrm{H}$ in specific is reported to increase the risk of late onset of Alzheimer's disease by two-fold. Not only does this variant increase the risk for Alzheimer's disease, it is also linked to Parkinson's disease, dementia, and sclerosis. After the TREM2 receptor is expressed on the cell membrane, it is cleaved by gamma-secretase. TREM2 is suggested to play an important role in neurodegeneration and clearing proteins through itself phagocytic pathway [18].

\section{Behavioral/Social}

\subsection{Educational Attainment \& Intellectual Activity}

The level of educational attainment and the amount of intellectual activity has been associated with the development of Alzheimer's disease. There have been many studies concluding that patients with Alzheimer's disease typically have lower amounts of intellectual activities throughout their lives. Results from one study, in particular, showed that low levels of educational attainment, as well as low participation in intellectual activities in midlife, is a risk factor for Alzheimer's disease. The study also reported people with less physical activity combined with a lack of intellectual activities had an increase in the risk of developing Alzheimer's disease by about 250\% [19].

Another study concluded similar results and also reported strong evidence of a causal, bidirectional relationship between intelligence and education attainment. It is theorized that the learning complex can increase synaptic connections in the gray matter of the brain and causes structural changes in the 
white matter which may contribute to increased intelligence. The findings of the study revealed increasing levels of educational attainment could reduce the incidence of Alzheimer's disease [20].

Given that many studies have concluded that educational attainment can play a role in reducing the risk of developing Alzheimer's disease, it is important for the public to be aware of this information. Countries like the UK have implemented changes in schooling legislation to mandate students to stay in school until the age of 18 . Possibly mandating higher education beyond the age of 18 could aid in reducing the incidence of Alzheimer's disease. However, if this is not a feasible option for everyone then governmental recommendations should suggest that people partake in regular cognitive training throughout life [20].

\subsection{Diet}

There has been some research showing that poor diet, such as high saturated fat and low vegetable intake, can increase the risk of Alzheimer's. There are a small number of studies on individual dietary components and data. However, there is increasing evidence showing that specific dietary practices, such as Mediterranean diet, can lower the risk of AD, while independent of physical activity and other comorbidities [21].

A Mediterranean diet consists of fish and plant foods, wine and olive oil as a source of monounsaturated fats, and low in red meat and poultry intake. The high amounts of antioxidants and polyunsaturated fatty acids in fruits, vegetables, and fish can reduce the incidence of AD as it has been found that reactive oxygen species is associated with neuronal damage in $A D[22,23]$.

As depositions of $A \beta$ increase, the levels of iron and copper reduce, resulting in oxidative and neuronal damage [24]. The intake of antioxidants can possibly decrease the risk of AD by lowering the risk of cerebrovascular disease [25].

While polyunsaturated fatty acids reduce oxidative stress and help with inflammatory processes and neuronal and vascular functions, some studies had inconclusive results regarding its impact and association with cognitive performance. On the other hand, in vitro studies have shown that $A \beta$-lipid peroxidation can be reduced by vitamin E, and carotenes and vitamin C can lower levels of lipid peroxidation and formation of nitrosamines. However, there are some inconclusive results about the impact of vitamin E and C [26-29].

As studies have tested the effects of cholesterol and saturated fats in relation to the development of $A D$, Van der Auwera et al. have shown the effects of a ketogenic diet (high saturated fats and low carbohydrates intake) on a transgenic mouse model. Mice fed the ketogenic diet had increased levels of ketone bodies, such as $\beta$-hydroxybutyrate, but lost body weight. Previous studies assessed the individual effect of high saturated fats which can increase the risk of AD. However, this study showed that a combined diet of lipid rich and low carbohydrates can decrease the total brain A $\beta$ levels [30].

The ketogenic diet has a very high-fat, low-carbohydrate content. It increases mitochondrial function and cellular metabolism, which may result in enhanced cognitive functioning in elderly patients with Alzheimer's Disease. The best results of KD treatment are expected in early presymptomatic stages of $A D$. This type of diet may cause hypoglycemia and dehydration [31]. 


\subsection{Physical Activity}

Some studies have shown that increases in physical activity have been linked to a reduction in the risk of developing Alzheimer's disease. Regular physical activity has been shown to decrease the risk of getting Alzheimer's disease by up to $31 \%$. Unlike other risk factors, this decrease is highly significant. This allows for additional interventions to reduce the risk of developing Alzheimer's disease. Additionally, this points to a modifiable lifestyle habit that has the ability to reduce the risk of AD. While the exact method cause of Alzheimer's disease is yet to be known, regular exercise allows for people genetically predisposed to AD to have a way to reduce their chances of developing AD [32-35].

A longitudinal study sought out to determine if leisure physical activity throughout midlife would have an effect on the risk of developing Alzheimer's disease and dementia. Patients were determined through questionnaires to evaluate their leisure-time physical time. After this information was recorded, participants were re-evaluated 21 years later. After multiple logistic regression, it was found that participants that incorporated leisure activity at least twice a week during their midlife had a reduced risk of dementia and Alzheimer's disease. The odds of getting Alzheimer's disease were $60 \%$ lower for those who exercised twice a week. The effects that physical activity had against dementia and Alzheimer's disease were more significant with APOE\&4 carriers, nevertheless, the study still showed significant results for non-carriers. The criteria for assessing leisure physical were if the participant participates in at least 20-30 minutes of physical activity that causes breathlessness and sweating. This could include anything from dancing to speed walking. The protective role that physical activity plays is rather significant and should be evaluated further as a possible way to prevent Alzheimer's disease. Simply adopting a more physically active lifestyle can go a long way in reducing the risk of Alzheimer's Disease [35].

\section{Existing Conditions}

\subsection{Hypertension}

Raised systolic blood pressure and hypercholesterolaemia in midlife can contribute to the onset of Alzheimer's disease. A longitudinal, population-based study showed that of 1449 people aging from 6579, 48 fulfilled the criteria for possible Alzheimer's. These patients were significantly older, had less formal education, and had a higher body mass index, serum cholesterol, and systolic blood pressure. However, no changes in diastolic blood pressure were observed. Participants with both high systolic pressure and serum cholesterol posed a higher risk of Alzheimer's and/or of myocardial infarction and cerebrovascular symptoms. Other studies have shown the risk can be related to raised diastolic blood pressure which can be due to the settings or populations of the studies. A study by Skoog et al. reported an increase in systolic and diastolic blood pressure 10-15 years prior to the onset of Alzheimer's disease [36].

Individuals with hypertension commonly exhibit an increase in senile plaques and neurofibrillary tangles [37].

Participants with Alzheimer's were likely to have received antihypertensive drugs in their midlife but have shown high systolic blood pressure later on. This signifies how high diastolic pressure is targeted 
for treatment, but even after receiving the treatment, they may not be able to achieve the desired blood pressure. Hypertension can also impact the blood brain barrier and cause protein extravasation into the brain tissue. This can result in damage to and apoptosis of cells, reduction of neuronal function, or beta-amyloid plaque accumulation which causes cognitive impairment [38].

Interestingly, with increasing age, the risk of elevated blood pressure for Alzheimer's disease decreases and can even provide a protective role. During the onset of Alzheimer's, weight loss, vessel stiffening, or changes in blood flow can consequently reduce blood pressure.

\subsection{Cholesterol}

Biochemical and epidemiological studies have shown a relation of $A \beta$-amyloid peptide, amyloid precursor protein, and cholesterol to Alzheimer's disease. Hypertension and hypercholesterolemia can cause atherosclerosis; impaired blood flow can contribute to the neurodegeneration seen in Alzheimer's disease. The combination of these two risk factors increases the risk for Alzheimer's [38].

Genetic linkage and association studies have reported certain genes involved in cholesterol transport or metabolism increase the susceptibility to Alzheimer's. These genes include ATP-binding cassette subfamily A member 7, apolipoprotein E, apolipoprotein J, and sortilin-related receptor [2].

Other studies have shown that cholesterol can alter beta-amyloid production by interfering with the process of beta-secretase and gamma-secretase enzymes acting upon beta-amyloid precursor. Additionally, cellular cholesterol also inhibits the colocalization of beta-secretase 1 and beta-amyloid precursor, resulting in a rise of plasmin, a beta-amyloid-degrading enzyme. Functional cell biology show that increased membrane cholesterol can be protective of the membrane due to amyloid cytotoxicity $[2,39]$.

In a study with 444 Finnish men aging from 70 to 89 years, high serum cholesterol levels of $\geq 6.5$ $\mathrm{mmol} / \mathrm{l}$ was associated with Alzheimer's disease. Factors such as apolipoprotein $\mathrm{E}$ gene and age were controlled. Of those who developed $A D$, a decrease in serum cholesterol levels before the symptoms of $A D$ appeared was observed, supporting cholesterol's protective role. In the Rotterdam study, it was found that there was no relation between serum cholesterol and dementia. However, a high dietary intake of saturated fat, total fat, and cholesterol increased the risk of both Alzheimer's and dementia. Intake of fish with n-3 polyunsaturated fatty acids decreased the risk of both dementia and Alzheimer's disease [40].

A study done by Sparks et al. showed that brains of rabbits with a high cholesterol diet had amyloid accumulations [41]. However, there are few studies to show the relation of cholesterol to Alzheimer's. A case control study based on a small hospital population showed Alzheimer's patients had higher mean cholesterol levels compared to controls. It was thought the apolipoprotein E gene impacted the cholesterol levels in these patients [42-44].

Based on a recent metanalysis study, the effect of $L D L$ cholesterol in development of $A D$ is important and higher level of LDL cholesterol in midlife is considered an independent risk factor for AD later in life. However, the effects of HDL cholesterol, total cholesterol and triglyceride levels were not statistically significant when compared with healthy control group [45]. 
The level of HDL cholesterol is controversial. Some studies indicate that lower level of HDL-C does not corelated with development of Alzheimer's disease [46-49]. However, some other studies indicate higher chance of developing AD if the patient suffers from lower level of HDL-C [50, 51].

\subsection{Diabetes Mellitus (DM)}

Diabetic patients have higher likelihood of developing dementia later in life [52, 53]. Risk factors for $D M$ and $A D$ somewhat tend to have similarities. Both conditions have inflammation, oxidative stress, and mitochondrial dysfunction. Brain insulin resistance and amyloidogenesis are crucial for hyperglycemia-induced deficiency of mental function [54].

It is suggested that hyperglycemia influences the neuronal damage and death which could eventually lead to a series of pathological changes. These pathological changes could induce amyloid plaque deposits leading to the development of Alzheimer's disease. Advanced glycation end products increase as people age; however, in diabetic patients, the rate of development of advanced glycation end products is accelerated. However, there is still much debate whether it plays a role in the development of Hirano bodies, neurofibrillary tangles, and beta-amyloid plaques which are indicative of Alzheimer's disease [55].

\subsection{Cardiovascular Disease (Homocysteine)}

It has been determined that elevated homocysteine levels are associated with atherosclerosis and an increase in the incidence of a stroke. Thus, the magnitude of increase in plasma homocysteine levels is correlated with an increased risk of death due to cardiovascular problems and the development of Alzheimer's disease. However, it has been established that an increase in homocysteine levels is also linked to Alzheimer's disease. An increase in plasma homocysteine levels from baseline by $5 \mu$ mol per liter increased the risk for the development of Alzheimer's disease by $40 \%$ [56].

In isolation of the resultant vascular consequences, a study evaluated cases where abnormally high levels of plasma homocysteine levels resulted in Alzheimer's disease without the presence of any vascular symptoms. Additionally, it was determined from a rat study that homocysteine promotes beta amyloid aggregation, in turn, inducing severe apoptosis in hippocampal cells [56].

Additional research points out that homocysteine and folate levels are inversely proportional to each other [57]. Hyperhomocysteinemia has been established to be a strong independent risk factor for Alzheimer's disease [56]. Therefore, efforts should be placed on detecting homocysteine levels before Alzheimer's disease develops in patients. It has been suggested that government-mandated folate and homocysteine be in place to reduce the incidence of AD. Efforts should be placed on early detection of $A D$ and should be furthered researched if decreasing homocysteine and increasing folate could prevent Alzheimer's disease [58].

\section{Conclusion}

Since Alzheimer's disease starts to progress years before symptoms appear, early detection and reversal is difficult. With $A D$ being one of the three leading causes of death in the older population, AD 
remains to be a complex series of changes in the brain, which is not fully understood by researchers as it is a multifactorial disease influenced by genetics, environment, and lifestyle. With genetics, there are multiple alleles and variants that play a role in the development of $A D$, and some AD-prone alleles can combine to produce an even more pronounced risk of AD. It is important to note that these identified alleles can have varied effects on specific populations depending on their demographics. Recent research has explored the impact that social and behavior modifications can have on the incidence of $A D$. Diets high in saturated fats with low vegetable intake can increase the risk of AD while following a Mediterranean diet has been associated with a lower risk of AD. Also, participating in regular physical exercise and engaging in intellectual activities has been seen to reduce the chances of developing AD. The comorbidity of some existing conditions and Alzheimer's disease can exacerbate the effects of AD on patients. However, some pre-existing conditions can actually play a protective role as the patient ages. Awareness about the many different actions people should take to help reduce their risk for AD should be spread as a public health initiative. $A D$ is a detrimental disease that has a significant impact on the quality of life of patients, thus further research should not only include exploring how $A D$ is caused, but also potential treatment options for AD should be investigated.

\section{Author Contributions}

All three authors contributed to different aspects of this paper.

\section{Competing Interests}

The authors have declared that no competing interests exist.

\section{References}

1. Parnetti L, Chipi E, Salvadori N, D’Andrea K, Eusebi P. Prevalence and risk of progression of preclinical Alzheimer's disease stages: A systematic review and meta-analysis. Alzheimers Res Ther. 2019; $11: 7$.

2. Reitz C, Mayeux R. Alzheimer disease: Epidemiology, diagnostic criteria, risk factors and biomarkers. Biochem Pharmacol. 2014; 88: 640-651.

3. Karch CM, Goate AM. Alzheimer's disease risk genes and mechanisms of disease pathogenesis. Biol Psychiatry. 2015; 77: 43-51.

4. Reitz C, Mayeux R. Genetics of Alzheimer's disease in Caribbean Hispanic and African American populations. Biol Psychiatry. 2014; 75: 534-541.

5. Chapuis J, Hansmannel F, Gistelinck M, Mounier A, Van Cauwenberghe C, Kolen KV, et al. Increased expression of BIN1 mediates Alzheimer genetic risk by modulating tau pathology. Mol Psychiatry. 2013; 18: 1225-1234.

6. Lambert JC, Heath S, Even G, Campion D, Sleegers K, Hiltunen M, et al. Genome-wide association study identifies variants at CLU and CR1 associated with Alzheimer's disease. Nat Genet. 2009; 41: 1094-1099. 
7. Meunier B, Quaranta M, Daviet L, Hatzoglou A, Leprince C. The membrane-tubulating potential of amphiphysin 2/BIN1 is dependent on the microtubule-binding cytoplasmic linker protein 170 (CLIP170). Eur J Cell Biol. 2009; 88: 91-102.

8. De Roeck A, Van Broeckhoven C, Sleegers K. The role of ABCA7 in Alzheimer's disease: Evidence from genomics, transcriptomics and methylomics. Acta Neuropathol. 2019; 138: 201-220.

9. Franz G, Beer R, Kampfl A, Engelhardt K, Schmutzhard E, Ulmer H, et al. Amyloid beta 1-42 and tau in cerebrospinal fluid after severe traumatic brain injury. Neurology. 2003; 60: 1457-1461.

10. Cruchaga C, Chakraverty S, Mayo K, Vallania FL, Mitra RD, Faber K, et al. Rare variants in APP, PSEN1 and PSEN2 increase risk for AD in late-onset Alzheimer's disease families. PloS ONE. 2012; 7: e31039.

11. Gerrish A, Russo G, Richards A, Moskvina V, Ivanov D, Harold D, et al. The role of variation at A $\beta P P$, PSEN1, PSEN2, and MAPT in late onset Alzheimer's disease. J Alzheimers Dis. 2012; 28: 377-387.

12. Kim M, Suh J, Romano D, Truong MH, Mullin K, Hooli B, et al. Potential late-onset Alzheimer's disease-associated mutations in the ADAM10 gene attenuate $\alpha$-secretase activity. Hum Mol Genet. 2009; 18: 3987-3996.

13. Bali J, Halima SB, Felmy B, Goodger Z, Zurbriggen S, Rajendran L. Cellular basis of Alzheimer's disease. Ann Indian Acad Neurol. 2010; 13: S89-S93.

14. Lichtenthaler SF. Alpha - secretase in Alzheimer's disease: Molecular identity, regulation and therapeutic potential. J Neurochem. 2011; 116: 10-21.

15. Zhong $\mathrm{N}$, Weisgraber $\mathrm{KH}$. Understanding the basis for the association of apoE4 with Alzheimer's disease: Opening the door for therapeutic approaches. Curr Alzheimer Res. 2009; 6: 415-418.

16. Brendza RP, Bales KR, Paul SM, Holtzman DM. Role of apoE/A $\beta$ interactions in Alzheimer's disease: Insights from transgenic mouse models. Mol Psychiatry. 2002; 7: 132-135.

17. Mayeux R, Ottman R, Maestre G, Ngai C, Tang MX, Ginsberg H, et al. Synergistic effects of traumatic head injury and apolipoprotein-epsilon4 in patients with Alzheimer's disease. Neurology. 1995; 45 : 555-557.

18. Karch CM, Cruchaga C, Goate AM. Alzheimer's disease genetics: From the bench to the clinic. Neuron. 2014; 83: 11-26.

19. Friedland RP, Fritsch T, Smyth KA, Koss E, Lerner AJ, Chen $\mathrm{CH}$, et al. Patients with Alzheimer's disease have reduced activities in midlife compared with healthy control-group members. Proc Natl Acad Sci U S A. 2001; 98: 3440-3445.

20. Anderson EL, Howe LD, Wade KH, Ben-Shlomo Y, Hill WD, Deary IJ, et al. Education, intelligence and Alzheimer's disease: Evidence from a multivariable two-sample Mendelian randomization study. Int J Epidemiol. 2020; 49: 1163-1172.

21. Scarmeas N, Stern Y, Mayeux R, Luchsinger JA. Mediterranean diet, Alzheimer disease, and vascular mediation. Arch Neurol. 2006; 63: 1709-1717.

22. Gu Y, Nieves JW, Stern Y, Luchsinger JA, Scarmeas N. Food combination and Alzheimer disease risk: A protective diet. Arch Neurol. 2010; 67: 699-706.

23. Scarmeas N, Stern Y, Mayeux R, Manly JJ, Schupf N, Luchsinger JA. Mediterranean diet and mild cognitive impairment. Arch Neurol. 2009; 66: 216-225. 
24. Nagano S, Huang X, Moir RD, Payton SM, Tanzi RE, Bush Al. Peroxidase activity of cyclooxygenase2 (COX-2) cross-links $\beta$-amyloid $(A \beta)$ and generates $A \beta$-COX-2 hetero-oligomers that are increased in Alzheimer's disease. J Biol Chem. 2004; 279: 14673-14678.

25. Voko Z, Hollander M, Hofman A, Koudstaal PJ, Breteler MM. Dietary antioxidants and the risk of ischemic stroke: The Rotterdam study. Neurology. 2003; 61: 1273-1275.

26. Allan Butterfield D, Castegna A, Drake J, Scapagnini G, Calabrese V. Vitamin E and neurodegenerative disorders associated with oxidative stress. Nutr Neurosci. 2002; 5: 229-239.

27. Pardo B, Mena MA, Fahn S, de Yébenes JG. Ascorbic acid protects against levodopa-induced neurotoxicity on a catecholamine-rich human neuroblastoma cell line. Mov Disord. 1993; 8: 278284.

28. Pitchumoni SS, Doraiswamy PM. Current status of antioxidant therapy for Alzheimer's disease. J Am Geriatr Soc. 1998; 46: 1566-1572.

29. Weisburger JH. Vitamin C and prevention of nitrosamine formation. Lancet. 1977; 2: 607.

30. Van der Auwera I, Wera S, Van Leuven F, Henderson ST. A ketogenic diet reduces amyloid beta 40 and 42 in a mouse model of Alzheimer's disease. Nutr Metab. 2005; 2: 28.

31. Rusek M, Pluta R, Ułamek-Kozioł M, Czuczwar SJ. Ketogenic diet in Alzheimer’s disease. Int J Mol Sci. 2019; 20: 3892.

32. Lindsay J, Laurin D, Verreault R, Hébert R, Helliwell B, Hill GB, et al. Risk factors for Alzheimer's disease: A prospective analysis from the Canadian study of health and aging. Am J Epidemiol. 2002; 156: 445-453.

33. Fratiglioni L, Paillard-Borg S, Winblad B. An active and socially integrated lifestyle in late life might protect against dementia. Lancet Neurol. 2004; 3: 343-353.

34. Lautenschlager NT, Cox KL, Flicker L, Foster JK, Van Bockxmeer FM, Xiao J, et al. Effect of physical activity on cognitive function in older adults at risk for Alzheimer disease: A randomized trial. JAMA. 2008; 300: 1027-1037.

35. Rovio S, Kåreholt I, Helkala EL, Viitanen M, Winblad B, Tuomilehto J, et al. Leisure-time physical activity at midlife and the risk of dementia and Alzheimer's disease. Lancet Neurol. 2005; 4: 705711.

36. Skoog I, Nilsson L, Persson G, Lernfelt B, Landahl S, Palmertz B, et al. 15-year longitudinal study of blood pressure and dementia. Lancet. 1996; 347: 1141-1145.

37. Serrano-Pozo A, Frosch MP, Masliah E, Hyman BT. Neuropathological alterations in Alzheimer disease. Cold Spring Harb Perspect Med. 2011; 1: a006189.

38. Kivipelto M, Helkala EL, Laakso MP, Hänninen T, Hallikainen M, Alhainen K, et al. Midlife vascular risk factors and Alzheimer's disease in later life: Longitudinal, population based study. BMJ. 2001; 322: 1447-1451.

39. Nicholson AM, Ferreira A. Cholesterol and neuronal susceptibility to beta-amyloid toxicity. Cogn Sci. 2010; 5: 35-56.

40. Notkola IL, Sulkava R, Pekkanen J, Erkinjuntti T, Ehnholm C, Kivinen P, et al. Serum total cholesterol, apolipoprotein E \{FC12\} e4 allele, and Alzheimer's disease. Neuroepidemiology. 1998; 17: 14-20.

41. Sparks DL. Cholesterol, copper, and accumulation of thioflavine S-reactive Alzheimer's-like amyloid $\beta$ in rabbit brain. J Mol Neurosci. 2004; 24: 97-104. 
42. Devassy JG, Leng S, Gabbs M, Monirujjaman M, Aukema HM. Omega-3 polyunsaturated fatty acids and oxylipins in neuroinflammation and management of Alzheimer disease. Adv Nutr. 2016; 7: 905916.

43. Devore EE, Grodstein F, van Rooij FJ, Hofman A, Rosner B, Stampfer MJ, et al. Dietary intake of fish and omega-3 fatty acids in relation to long-term dementia risk. Am J Clin Nutr. 2009; 90: 170-176.

44. Kalmijn S, Launer LJ, Ott A, Witteman JC, Hofman A, Breteler MM. Dietary fat intake and the risk of incident dementia in the Rotterdam study. Ann Neurol. 1997; 42: 776-782.

45. Sáiz-Vazquez O, Puente-Martínez A, Ubillos-Landa S, Pacheco-Bonrostro J, Santabárbara J. Cholesterol and Alzheimer's disease risk: A meta-meta-analysis. Brain Sci. 2020; 10: 386.

46. Liu Y, Zhong X, Shen J, Jiao L, Tong J, Zhao W, et al. Elevated serum TC and LDL-C levels in Alzheimer's disease and mild cognitive impairment: A meta-analysis study. Brain Res. 2020; 1727: 146554.

47. Wu Y, Wang Z, Jia X, Zhang H, Zhang H, Li J, et al. Prediction of Alzheimer's disease with serum lipid levels in Asian individuals: A meta-analysis. Biomarkers. 2019; 24: 341-351.

48. Xu W, Tan L, Wang HF, Jiang T, Tan MS, Tan L, et al. Meta-analysis of modifiable risk factors for Alzheimer's disease. J Neurol Neurosurg Psychiatry. 2015; 86: 1299-1306.

49. Tan ZS, Seshadri S, Beiser A, Wilson PW, Kiel DP, Tocco M, et al. Plasma total cholesterol level as a risk factor for Alzheimer disease: The Framingham study. Arch Intern Med. 2003; 163: 1053-1057.

50. Carleton RA, Dwyer J, Finberg L, Flora J, Goodman DS, Grundy SM, et al. Report of the expert panel on population strategies for blood cholesterol reduction. A statement from the national cholesterol education program, national heart, lung, and blood institute, national institutes of health. Circulation. 1991; 83: 2154-2232.

51. Reitz C, Tang MX, Luchsinger J, Mayeux R. Relation of plasma lipids to Alzheimer disease and vascular dementia. Arch Neurol. 2004; 61: 705-714.

52. Irie F, Fitzpatrick AL, Lopez OL, Kuller LH, Peila R, Newman AB, et al. Enhanced risk for Alzheimer disease in persons with type 2 diabetes and APOE $\varepsilon 4$ : The cardiovascular health study cognition study. Arch Neurol. 2008; 65: 89-93.

53. Rönnemaa E, Zethelius B, Sundelöf J, Sundström J, Degerman-Gunnarsson M, Berne C, et al. Impaired insulin secretion increases the risk of Alzheimer disease. Neurology. 2008; 71: 1065-1071.

54. Lee HJ, Seo HI, Cha HY, Yang YJ, Kwon SH, Yang SJ. Diabetes and Alzheimer's disease: Mechanisms and nutritional aspects. Clin Nutr Res. 2018; 7: 229-240.

55. Breteler MM. Vascular risk factors for Alzheimer's disease: An epidemiologic perspective. Neurobiol Aging. 2000; 21: 153-160.

56. Seshadri S, Beiser A, Selhub J, Jacques PF, Rosenberg IH, D'Agostino RB, et al. Plasma homocysteine as a risk factor for dementia and Alzheimer's disease. N Engl J Med. 2002; 346: 476-483.

57. Clarke R, Smith AD, Jobst KA, Refsum H, Sutton L, Ueland PM. Folate, vitamin B12, and serum total homocysteine levels in confirmed Alzheimer disease. Arch Neurol. 1998; 55: 1449-1455.

58. Morris MS. Homocysteine and Alzheimer's disease. Lancet Neurol. 2003; 2: 425-428. 
OBM Neurobiology 2021; 5(3), doi:10.21926/obm.neurobiol.2103104

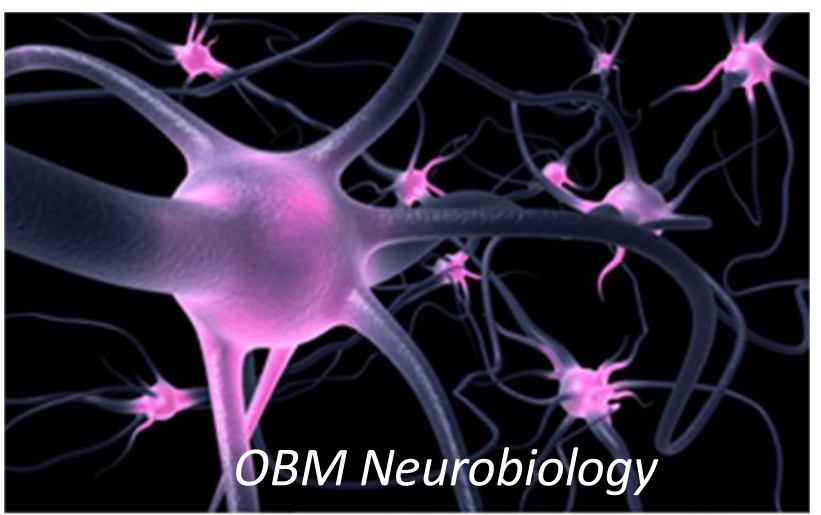

Enjoy OBM Neurobiology by:

1. Submitting a manuscript

2. Joining volunteer reviewer bank

3. Joining Editorial Board

4. Guest editing a special issue For more details, please visit:

http://www.lidsen.com/journals/neurobiology 